ANNALES

POLONICI MATHEMATICI

$98.3(2010)$

\title{
The Abhyankar-Jung theorem for excellent henselian subrings of formal power series
}

\author{
by KRZYSZTof JAN NOWAK (Kraków)
}

\begin{abstract}
Given an algebraically closed field $K$ of characteristic zero, we prove the Abhyankar-Jung theorem for any excellent henselian ring whose completion is a formal power series ring $K[[z]]$. In particular, examples include the local rings which form a Weierstrass system over the field $K$.
\end{abstract}

The Abhyankar-Jung theorem may be regarded as a higher dimensional counterpart of the Newton-Puiseux theorem. It asserts that the roots of a Weierstrass (formal or convergent) polynomial over an algebraically closed field of characteristic zero with discriminant being a normal crossing are fractional (formal or convergent) series. The first proof for the case of two complex variables was due to H. W. Jung [6]. The general result for the algebroid case (for several variables and an arbitrary ground field) was established by S. S. Abhyankar [1] by means of purely algebraic methods, namely, some properties of the Galois group of the polynomial under study. The methods of Jung and Abhyankar are described e.g. in [8].

The classical proofs of the Newton-Puiseux theorem applied Newton's algorithm to compute, term by term, the fractional series (called Puiseux series) arising as $t$-roots of an algebraic equation $f(x, t)=0$. This algorithm, invented in "Methodus fluxionum et serierum infinitorum" (see [11], and also [19]), uses the so-called Newton polygon, determined by the exponents of a given polynomial.

More recently, I. Luengo [9] deduced the Abhyankar-Jung theorem by analyzing, by analogy with the one variable case, the Newton polyhedron of the polynomial under study. This was done by applying his statement that a quasiordinary formal Weierstrass polynomial $f(x ; t) \in K[[x]][t]$ with vanishing coefficient of $t^{n-1}, n=\operatorname{deg} f$, is $\nu$-quasiordinary in the sense of

2010 Mathematics Subject Classification: 13F25, 13F40.

Key words and phrases: Abhyankar-Jung theorem, excellent henselian rings, formal power series. 
Hironaka [5]; the latter is a certain property of the Newton polyhedron. However, his proof of this statement seems to contain — as indicated in [7]an essential gap. Paper [7] by K. Kiyek and J. L. Vicente was intended to provide a proof for both the formal and convergent cases in a language scholarly acceptable today. Their approach, relying heavily on ramification theory (as developed by A. Grothendieck in [4]), followed an idea taken from Zariski's paper [23].

In our paper, we generalize the Abhyankar-Jung theorem to the case of any excellent henselian ring whose completion is a power series ring over an algebraically closed field $K$ of characteristic zero; this covers the classical ones of formal and convergent series. The proofs we present here are very short. In the convergent case, we use a well-known lifting criterion for unramified covers and the Riemann removable singularity theorem (cf. our topological proof of Puiseux's theorem in [12]). The passage upwards, to the formal power series ring, and next downwards, to an excellent henselian subring, is attained here by means of the Artin approximation theorem. In our approach, an arbitrary algebraically closed field $K$ of characteristic zero is regarded as an o-minimal expansion of a maximal real subfield $R$ of $K$. Due to the theory developed by Y. Peterzil and S. Starchenko [17, 18, the field $K$ over $R$ can be treated, in many respects, in the same way as the complex field $\mathbb{C}$ over the real field $\mathbb{R}$. Often the results about $K$-differentiability are even stronger than the classical ones, because the topology of definable sets is tame. However, techniques concerning convergent power series and analytic prolongation are unavailable.

In our subsequent papers [14, 15], we shall apply the version of the Abhyankar-Jung theorem presented here to o-minimal structures determined by a convergent Weierstrass system.

From now on $K$ denotes an algebraically closed field of characteristic zero and $R$ a maximal real subfield of $K$; obviously, $R$ is a real closed field and $K=R[\sqrt{-1}]$. As in the classical case, we may identify $K$ with $R^{2}$ and equip it with the product topology inherited from the ordered field $R$. Given any o-minimal expansion $\mathcal{R}$ of the real closed field $R$, one can develop, similarly to the case of ordinary holomorphic functions, the theory of $K$ differentiable functions definable in the structure $\mathcal{R}$. Observe that in the case of a polynomial, the $K$-derivatives and the formal derivatives in the polynomial ring coincide, and thus the notion of derivative for polynomial mappings is independent of the initial choice of the maximal real subfield $R$.

The analogues of the most classical results, including the CauchyRiemann equations, Riemann removable singularity theorem, maximum principle as well as the Weierstrass preparation and division theorems, have been established by Y. Peterzil and S. Starchenko [17, 18]. Consequently, the 
ring $K\langle z\rangle, z=\left(z_{1}, \ldots, z_{m}\right)$, of $K$-differentiable $\mathcal{R}$-definable function germs at $0 \in K^{m}$ is a noetherian local ring with maximal ideal generated by the $m$ coordinates, and its completion is the formal power series ring $K[[z]]$. Moreover, the ring $K\langle z\rangle$ is regular, henselian and excellent (by a Jacobian criterion; cf. [10, Section 40, Theorem 102]). In this fashion, a major part of the classical complex analytic geometry can be carried over to the $K$ differentiable setting. In particular, we have at our disposal $K$-differentiable analogues of the results concerning ramified and unramified analytic coverings. Their proofs can be repeated mutatis mutandis; for instance, the topological notions such as "neighbourhood", "connected", "compact", "proper" etc., have to be replaced by "definable neighbourhood", "definably connected", "definably compact", "definably proper" etc.

We call a polynomial

$$
f(z ; t)=t^{n}+a_{1}(z) t^{n-1}+\cdots+a_{n}(z) \in K[[z]][t], \quad z=\left(z_{1}, \ldots, z_{m}\right),
$$

quasiordinary if its discriminant $D(z)$ is a normal crossing:

$$
D(z)=z^{\gamma} \cdot u(z) \quad \text { with } \gamma \in \mathbb{N}^{m}, u(z) \in K[[z]], u(0) \neq 0 .
$$

We say that $f(z ; t)$ is a Weierstrass polynomial if its coefficients $a_{i}(z)$ belong to the maximal ideal of $K[[z]]$, i.e. $a_{k}(0)=0$. For any integers $r_{1}, \ldots, r_{m}>0$, put

$$
K\left\langle z_{1}^{1 / r_{1}}, \ldots, z_{m}^{1 / r_{m}}\right\rangle:=\left\{\alpha\left(z_{1}^{1 / r_{1}}, \ldots, z_{m}^{1 / r_{m}}\right): \alpha(z) \in K\langle z\rangle\right\} ;
$$

when $r_{1}=\cdots=r_{m}=r$, we shall denote the above $K$-algebra by $K\left\langle z^{1 / r}\right\rangle$. Now we can readily establish a $K$-differentiable version of

Abhyankar-Jung Theorem. Every quasiordinary polynomial

$$
f(z ; t)=t^{n}+a_{1}(z) t^{n-1}+\cdots+a_{n}(z) \in K\langle z\rangle[t]
$$

has all its roots in $K\left\langle z^{1 / r}\right\rangle$ for some $r \in \mathbb{N}$; actually one can take $r=n$ !.

To see this, since the ring $K\langle z\rangle$ is henselian, we may assume that $f(z ; t)$ is a Weierstrass polynomial. We may also suppose $f(z ; t)$ is irreducible. As we have already outlined, each ring $K\langle z\rangle[t]$ under consideration can be treated exactly in the same way as in the classical complex analytic geometry. Therefore, without loss of generality, we may confine ourselves to the case of the ring of convergent complex power series. For simplicity, we shall denote germs and their representatives in a common neighbourhood $U \subset \mathbb{C}^{m}$ by the same symbols. We may take $U$ to be a polydisk $B_{\epsilon}^{m}$ with $B_{\epsilon}=\{c \in \mathbb{C}:|c|<\epsilon\}$, $\epsilon>0$. Let

$$
V:=\{(z, t) \in U \times \mathbb{C}: f(z ; t)=0\}
$$

be the zero locus of the polynomial $f(z ; t)$, and

$$
p: \mathbb{C}_{z}^{m} \times \mathbb{C}_{t} \rightarrow \mathbb{C}_{z}^{m}, \quad p(z, t)=z,
$$


the canonical projection. Then $S:=\{z \in U: D(z)=0\}$ is the ramification divisor of the ramified analytic covering res $p: V \rightarrow U$. Since $D(z)$ is a normal crossing, $U \backslash S \supset\left(B_{\epsilon}^{*}\right)^{m}$ with $B_{\epsilon}^{*}:=B_{\epsilon} \backslash\{0\}$. Denote by $Z$ the cross $U \backslash\left(B_{\epsilon}^{*}\right)^{m}$. Then $S \subset Z$ and

$$
\operatorname{res} p:\left(V \backslash p^{-1}(Z)\right) \rightarrow\left(B_{\epsilon}^{*}\right)^{m}
$$

is a proper unramified covering. Clearly, the group

$$
p_{*}\left(\pi_{1}\left(V \backslash p^{-1}(Z)\right)\right) \subset \pi_{1}\left(\left(B_{\epsilon}^{*}\right)^{m}\right)=\mathbb{Z}^{m}
$$

contains $r \cdot \mathbb{Z}^{m}$ for some integer $r>0$; here $\pi_{1}(E)$ denotes the first homotopy group of an arc-connected set $E$. Put $\delta:=\epsilon^{1 / r}$ and

$$
\varphi:\left(B_{\delta}^{*}\right)^{m} \rightarrow\left(B_{\epsilon}^{*}\right)^{m}, \quad \varphi(z):=z^{r}=\left(z_{1}^{r}, \ldots, z_{m}^{r}\right) .
$$

Then we have

$$
\varphi_{*}\left(\pi_{1}\left(\left(B_{\delta}^{*}\right)^{m}\right)\right) \subset p_{*}\left(\pi_{1}\left(V \backslash p^{-1}(Z)\right)\right) .
$$

By a well-known lifting criterion (see e.g. [3, p. 20]), there is a continuous mapping

$$
G:\left(B_{\delta}^{*}\right)^{m} \rightarrow V \backslash p^{-1}(Z)
$$

such that $p \circ G=\varphi$. It is easy to check that $G$ is a holomorphic mapping of the form $G(z)=\left(z^{r}, g(z)\right)$. Further, the mapping $G$ is open and proper, hence closed, and thus surjective, because the set $V \backslash p^{-1}(Z)$ is connected. Since $G$ is a bounded mapping on $\left(B_{\delta}^{*}\right)^{m}$, it extends - by virtue of the Riemann removable singularity theorem - to a surjective holomorphic mapping $G$ : $B_{\delta}^{m} \rightarrow V$. Hence

$$
V=\left\{\left(z^{r}, g(z)\right): z \in B_{\delta}^{m}\right\} \quad \text { or equivalently } \quad V=\left\{\left(z, g\left(z^{1 / r}\right)\right): z \in B_{\epsilon}^{m}\right\},
$$

where $z_{i}^{1 / r}$ denotes a multi-valued function. This proves the first part of the theorem.

Finally, since res $p:\left(V \backslash p^{-1}(Z)\right) \rightarrow\left(B_{\epsilon}^{*}\right)^{m}$ is an $n$-fold covering, we actually get

$$
r_{1} \cdot \mathbb{Z} \oplus \cdots \oplus r_{m} \cdot \mathbb{Z} \subset p_{*}\left(\pi_{1}\left(V \backslash p^{-1}(Z)\right)\right)
$$

for some positive integers $r_{1}, \ldots, r_{m} \leq n$. We may thus take $r$ to be the least common multiple of $r_{1}, \ldots, r_{m}$, concluding the proof of the theorem.

Let us mention that for the one-dimensional version of the theorem, i.e., the Newton-Puiseux theorem, the estimate of $r$ can be deduced from the algebraic proofs as well (e.g. the one given in [13]).

REMARK 1. Suppose that the discriminant $D(z)$ of the polynomial $f(z ; t)$ is a normal crossing of the form

$$
D(z)=z_{1}^{\gamma_{1}} \cdots z_{p}^{\gamma_{p}} \cdot u(z) \text { with } \quad u(0) \neq 0,0 \leq p \leq m .
$$


Then, considering the restrictions of the polynomial $f(z ; t)$ to the affine lines parallel to the coordinate axes, we see that

$$
\left(r \cdot \mathbb{Z}^{p}\right) \oplus \mathbb{Z}^{m-p} \subset p_{*}\left(\pi_{1}\left(V \backslash p^{-1}(S)\right)\right) .
$$

Consequently, all the roots of the polynomial $f(z ; v)$ belong to the ring $K\left\langle z_{1}^{1 / r}, \ldots, z_{p}^{1 / r}, z_{p+1}, \ldots, z_{m}\right\rangle$.

Going upwards, we shall prove the Abhyankar-Jung theorem for the formal power series rings. So let $K$ be an algebraically closed field of characteristic zero and

$$
f(z ; t)=t^{n}+a_{1}(z) t^{n-1}+\cdots+a_{n}(z) \in K[[z]][t]
$$

be a quasiordinary Weierstrass polynomial with discriminant $D(z)=z^{\gamma}$. $u(z), u(0) \neq 0$. Take a maximal real subfield $R$ of $K$ and the o-minimal structure $\mathcal{R}=(R,<, 0,1,+,-, \cdot)$, and consider the ring $K\langle z\rangle, z=\left(z_{1}, \ldots, z_{m}\right)$, of $K$-differentiable $\mathcal{R}$-definable function germs at $0 \in K^{m}$. Since $K\langle z\rangle$ is an excellent henselian ring, it has the Artin approximation property (cf. [21, 22]). Therefore the polynomial $f(z ; t)$ can be approximated in the Krull topology by a sequence of quasiordinary Weierstrass polynomials $f_{k}(z ; t) \in K\langle z\rangle[t]$. Indeed, the discriminant $D(z)$ of the polynomial $f(z, t)$ can be expressed as

$$
D(z)=P\left(a_{1}(z), \ldots, a_{n}(z)\right) \quad \text { where } P\left(T_{1}, \ldots, T_{n}\right) \in \mathbb{Z}\left[T_{1}, \ldots, T_{n}\right] .
$$

Our assertion thus follows from the Artin approximation theorem applied to the system of two polynomial equations

$$
P\left(A_{1}, \ldots, A_{n}\right)=z^{\gamma} \cdot U, \quad U \cdot V=1,
$$

with indeterminates $A_{1}, \ldots, A_{n}, U, V$.

Now, by the $K$-differentiable version of the Abhyankar-Jung theorem, each polynomial $f_{k}(z ; t)$ has all its roots in $K\left\langle z^{1 / r}\right\rangle[t]$ with $r=n$ !. Therefore, the proof will be completed once we know that the roots depend continuously in the Krull topology on the coefficients. But this follows immediately from the corollary to a valuation theorem on the continuity of roots (cf. 2, Section 4, Theorem 2]; see also [20, Proposition 7]), recalled in the proposition below. Indeed, we apply this corollary to the valuation of the quotient field of the ring $K\left[\left[z^{1 / r}\right]\right], r=n$ !, which extends the order function.

Proposition. Let $(F, v)$ be a valued field with value group $\Gamma$. Consider two monic polynomials of degree $n$ :

$$
f(t)=t^{n}+a_{1} t^{n-1}+\cdots+a_{n}, \quad g(t)=t^{n}+b_{1} t^{n-1}+\cdots+b_{n}
$$

with integral coefficients $a_{i}, b_{i} \in F$ (i.e. $v\left(a_{i}\right), v\left(b_{i}\right) \geq 0$ for $i=1, \ldots, n$ ). 
Suppose that

$$
f(t)=\prod_{i=1}^{n}\left(t-\alpha_{i}\right) \quad \text { and } \quad g(t)=\prod_{i=1}^{n}\left(t-\beta_{i}\right),
$$

where $\alpha_{i}, \beta_{i} \in F, i=1, \ldots, n$. Define a strictly increasing error function $\Phi_{j}(\gamma)$ of the root $\alpha_{j}$ of $f(t)$ by putting

$$
\Phi_{j}: \Gamma \cap\{\infty\} \rightarrow \Gamma \cap\{\infty\}, \quad \Phi_{j}(\gamma):=\sum_{i=1}^{n} \min \left\{\gamma, v\left(\alpha_{j}-\alpha_{i}\right)\right\} .
$$

Then, after performing a suitable permutation of the roots, we have

$$
\Phi_{j}\left(v\left(\alpha_{j}-\beta_{j}\right)\right) \geq v(f-g), \quad j=1, \ldots, n,
$$

where, for a polynomial $h \in F[t], v(h)$ stands for the minimum of the values of its coefficients.

The proof of this proposition consists in analyzing the valuation Newton polygons of the two polynomials under study. We wish to emphasize that whereas it is usually difficult to determine the roots by means of the coefficients, the computation of the values of the roots by means of the values of the coefficients is quite easy.

COROLlary. Under the above assumptions, we have

$$
n \cdot v\left(\alpha_{j}-\beta_{j}\right) \geq v(f-g), \quad j=1, \ldots, n,
$$

after performing a suitable permutation of the roots.

In our case, however, the conclusion of this corollary can be obtained by yet another, even simpler, reasoning. Indeed, let $\alpha_{i, k}(z) \in K\left\langle z^{1 / r}\right\rangle, i=$ $1, \ldots, n$, be the roots of the polynomials $f_{k}(z ; t), k \in \mathbb{N}$. Since the discriminants of all polynomials $f_{k}(z ; t)$ are of the form $D_{k}(z)=z^{\gamma} \cdot u_{k}(z)$ with $u_{k}(0) \neq 0$, we get

$$
\operatorname{ord}\left(\alpha_{i, k}-\alpha_{j, k}\right) \leq \gamma \quad \text { for all } k \in \mathbb{N}, 1 \leq i, j \leq n, i \neq j .
$$

Therefore, our assertion follows from an elementary

Claim. Under the assumptions of the foregoing proposition, for each root $\alpha_{i}$ of $f(t)$ there is a root $\beta_{j}$ of $g(t)$ such that $n \cdot v\left(\alpha_{i}-\beta_{j}\right) \geq v(f-g)$.

To prove the claim, take any root $\alpha=\alpha_{i}$. It is not difficult to check that

$$
v(\alpha) \geq \min \left\{v\left(a_{i}\right) / i: i=1, \ldots, n\right\} \geq 0 .
$$

Clearly,

$$
\prod_{j=1}^{n}\left(\alpha-\beta_{j}\right)=g(\alpha)=g(\alpha)-f(\alpha)=\left(b_{1}-a_{1}\right) \alpha^{n-1}+\left(b_{2}-a_{2}\right) \alpha^{n-2}+\cdots+\left(b_{n}-a_{n}\right),
$$


and thus

$$
\begin{aligned}
\sum_{j=1}^{n} v\left(\alpha-\beta_{j}\right) & \geq \min \left\{v\left(b_{1}-a_{1}\right)+(n-1) v(\alpha), \ldots, v\left(b_{n}-a_{n}\right)\right\} \\
& \geq \min \left\{v\left(b_{1}-a_{1}\right), \ldots, v\left(b_{n}-a_{n}\right)=: v(f-g) .\right.
\end{aligned}
$$

Hence $n \cdot v\left(\alpha-\beta_{j}\right) \geq v(f-g)$ for some $j=1, \ldots, n$, as asserted.

At this stage we can turn to the Abhyankar-Jung theorem for any excellent henselian ring whose completion is a formal power series ring over $K$, stated below.

MAIN THEOREM. Let $K$ be an algebraically closed field of characteristic zero, and $K\langle z\rangle$ be an excellent henselian ring whose completion is a power series ring $K[[z]], z=\left(z_{1}, \ldots, z_{m}\right)$. Then every quasiordinary polynomial

$$
f(z ; t)=t^{n}+a_{1}(z) t^{n-1}+\cdots+a_{n}(z) \in K\langle z\rangle[t]
$$

has all its roots in

$$
K\left\langle z^{1 / r}\right\rangle:=\left\{\alpha\left(z^{1 / r}\right): \alpha(z) \in K\langle z\rangle\right\}
$$

for some $r \in \mathbb{N}$; actually one can take $r=n !$.

Our proof will again make use of the Artin approximation property. By virtue of the Abhyankar-Jung theorem for formal power series, all $n$ roots of the polynomial $f(z ; t)$ can be represented in the following form:

$$
\alpha_{i}(z)=\sum_{\gamma_{1}, \ldots, \gamma_{m}=0}^{r-1} \alpha_{i, \gamma}(z) \cdot z^{\gamma / r}, \quad i=1, \ldots, n,
$$

where $r=n$ !. After substitution of the term

$$
A=\sum_{\gamma_{1}, \ldots, \gamma_{m}=0}^{r-1} A_{\gamma} \cdot z^{\gamma / r}
$$

for the indeterminate $t$ in the polynomial $f(z ; t)$, we get $r^{m}$ polynomial equations in the indeterminates $A_{\gamma}$ with coefficients from $K\langle z\rangle$; those coefficients are linear combinations of the products of the $a_{i}(z)$ 's by some powers of the variables z. Clearly,

$$
\left(\alpha_{1, \gamma}(z)\right)_{\gamma}, \ldots,\left(\alpha_{n, \gamma}(z)\right)_{\gamma}
$$

are the sole $n$ solutions to this system of polynomial equations. Hence and by the Artin approximation theorem, the components of these $n$ solutions belong to the ring $K\langle z\rangle$, as required.

REMARK 2. We have proven, in fact, that all roots of the polynomial $f(z ; t)$ belong to

$$
\sum_{\gamma_{1}, \ldots, \gamma_{m}=0}^{r-1} K\langle z\rangle \cdot z^{\gamma / r}
$$


Corollary (Real version of the Abhyankar-Jung theorem). Let $R$ be a real closed field and $R\langle x\rangle$ an excellent henselian ring whose completion is a power series ring $R[[x]], x=\left(x_{1}, \ldots, x_{m}\right)$. Then every quasiordinary polynomial

$$
f(x ; t)=t^{n}+a_{1}(x) t^{n-1}+\cdots+a_{n}(x) \in K\langle x\rangle[t]
$$

has a factorization of the form

$$
f(x ; t)=\prod_{i=1}^{p}\left(t-\varphi_{i}\left(x^{1 / r}\right)\right) \prod_{j=1}^{q}\left(t^{2}-\alpha_{j}\left(x^{1 / r}\right) t+\beta_{j}\left(x^{1 / r}\right)\right),
$$

where $r \in \mathbb{N}, p+2 q=n, \varphi_{i}, \alpha_{j}, \beta_{j} \in R\langle x\rangle$; actually one can take $r=n$ !.

Indeed, it suffices to apply the theorem to the excellent henselian ring $R\langle x\rangle \otimes_{R} R(\sqrt{-1})$ whose completion is $R(\sqrt{-1})[[x]]$.

REMARK 3 . The foregoing results apply to the ring of quasianalytic germs in one variable since it is a discrete valuation ring. Therefore, a counterpart of the Newton-Puiseux theorem is valid in the quasianalytic setting.

We conclude this paper with the following comment. In [16], we generalized the Abhyankar-Jung theorem-making use of Luengo's statement from [9] that every quasiordinary Weierstrass polynomial in the Tschirnhausen form is $\nu$-quasiordinary in the sense of Hironaka- to the case of henselian $k[x]$-algebras of formal power series, which are closed under reciprocal, power substitution and division by a coordinate. This allowed us to carry over that theorem to the local rings of quasianalytic function germs in several variables in polynomially bounded o-minimal structures. However, those results of ours bear a relative character, because it turned out, as already mentioned, that Luengo's proof seems to have an essential gap.

Acknowledgements. This research was partially supported by Research Project No. N N201 372336 from the Polish Ministry of Science and Higher Education.

\section{References}

[1] S. S. Abhyankar, On the ramification of algebraic functions, Amer. J. Math. 77 (1955), 575-592.

[2] D. Brink, New light on Hensel's lemma, Expo. Math. 24 (2006), 291-306.

[3] M. J. Greenberg, Lectures on Algebraic Topology, Benjamin, New York, 1967.

[4] A. Grothendieck, Éléments de Géométrie Algébrique, Vol. IV, Publ. Math. I.H.E.S. No. 20, 24, 28, 32, 1964-1967.

[5] H. Hironaka, Introduction to the theory of infinitely near singular points, Memorias Mat. Inst. "Jorge Juan" 28, C.S.I.C., Madrid, 1974. 
[6] H. W. E. Jung, Darstellung der Funktionen eines algebraischen Körpers zweier unabhängigen Veränderlichen $x, y$ in der Umgebung einer Stelle $x=a, y=b$, J. Reine Angew. Math. 133 (1908), 289-314.

[7] K. Kiyek and J. L. Vicente, On the Jung-Abhyankar theorem, Arch. Math. (Basel) 83 (2004), 123-134.

[8] J. Lipman, Introduction to resolution of singularities, in: Algebraic Geometry (Arcata, 1974), Proc. Sympos. Pure Math. 29, Amer. Math. Soc., 1975, 187-230.

[9] I. Luengo, A new proof of the Jung-Abhyankar theorem, J. Algebra 85 (1983), 399409.

[10] H. Matsumura, Commutative Algebra, Benjamin/Cummings, Reading, 1980.

[11] I. Newton, The Mathematical Papers of Isaac Newton, Vol. III: 1670-1673, ed. by D. T. Whiteside, Cambridge Univ. Press, London, 1969.

[12] K. J. Nowak, A simple proof of Puiseux's theorem, Univ. Iagel. Acta Math. 32 (1995), 199-200.

[13] - Some elementary proofs of Puiseux's theorems, ibid. 38 (2000), 279-282.

[14] - Rectilinearization of functions definable by a Weierstrass system and its applications, Ann. Polon. Math., to appear.

[15] -, On arc-analytic functions definable by a Weierstrass system, ibid., to appear.

[16] - On the Abhyankar-Jung theorem for henselian $k[x]$-algebras of formal power series, IMUJ Preprint 2, 2009.

[17] Y. Peterzil and S. Starchenko, Expansions of algebraically closed fields in o-minimal structures, Selecta Math. (N.S.) 7 (2001), 409-445.

[18] - - - Expansions of algebraically closed fields II: functions of several variables, J. Math. Logic 3 (2003), 1-35.

[19] V. Puiseux, Recherches sur les fonctions algébriques, J. Math. Pures Appl. 15 (1850), 365-480.

[20] P. Ribenboim, Théorie des Valuations, Presses Univ. de Montréal, Montreal, 1968.

[21] C. Rotthaus, On the approximation property of excellent rings, Invent. Math. 88 (1987), 39-63.

[22] -, Rings with approximation property, Math. Ann. 287 (1990), 455-466.

[23] O. Zariski, On the purity of the branch locus of algebraic functions, Proc. Nat. Acad. Sci. U.S.A. 44 (1958), 791-796.

Krzysztof Jan Nowak

Institute of Mathematics

Jagiellonian University

Łojasiewicza 6

30-348 Kraków, Poland

E-mail: nowak@im.uj.edu.pl

Received 11.2.2009

and in final form 8.4.2010 
